\title{
Reconstruction of Hadronically Decaying Tau Leptons with ATLAS
}

\author{
Blake Burghgrave* \\ Northern Illinois University \\ E-mail: blake.burghgrave@cern.ch
}

\section{On behalf of the ATLAS Collaboration}

Tau leptons play an important role in many physics processes in the Standard Model and beyond that are being investigated at the LHC. We summarize the reconstruction, identification and energy calibration algorithms for hadronic tau decays employed to analyze the data collected from proton-proton collisions in 2015-2016 running of the LHC at the center-of-mass energy of $\sqrt{s}=13 \mathrm{TeV}$. The algorithms are stable with respect to the number of concurrent proton-proton interactions and have been used in a variety of physics analyses by ATLAS. The performance is measured in most cases with $Z$ boson decays to tau lepton pairs selected from the collision data.

38th International Conference on High Energy Physics 3-10 August 2016

Chicago, USA

${ }^{*}$ Speaker. 


\section{Introduction}

We discuss the reconstruction, identification, energy calibration, and performance of hadronically decaying tau leptons by the ATLAS experiment [1].

Tau $(\tau)$ leptons are the only flavor of lepton massive enough to decay hadronically, with $m_{\tau}=1.78 \mathrm{GeV}$. Since the proper decay length is only 87 microns, taus decay well before reaching the detector, so they must be reconstructed from their decay products. Hadronic decays produce a narrow energy deposit (with respect to QCD) in the calorimeter, associated with low track multiplicity (generally 1- or 3-"prongs", or tracks from charged pions) and 0 or more neutral constituents, with significant missing transverse energy carried away by the neutrino. Therefore, we can directly measure only the hadronic component, referred to as $\tau_{\text {had-vis }}$.

\section{Reconstruction}

Hadronic tau reconstruction is seeded by an anti- $k_{t}$ jet with $\Delta R=0.4,|\eta|<2.5$, and $p_{\mathrm{T}}>$ $10 \mathrm{GeV}$. In events with pileup, the default primary vertex does not always correspond to the vertex at which the tau lepton was produced. The Tau Vertex Association algorithm uses tracks in a $\Delta R=0.2$ cone around the seed jet direction to identify the primary vertex associated with the tau. The $p_{\mathrm{T}}$ of the tracks is summed, and the vertex with the largest fraction of the sum is selected as the tau vertex. The tau vertex is used to determine the direction of the visible tau decay products, define the coordinate system used to recalculate impact parameters and identification variables, and perform track selection.

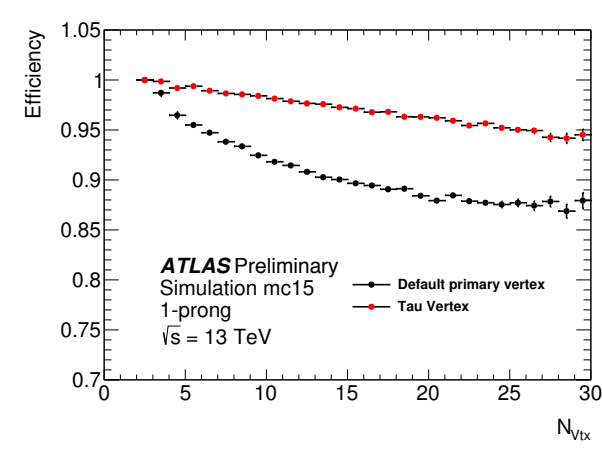

(a) Vertex association

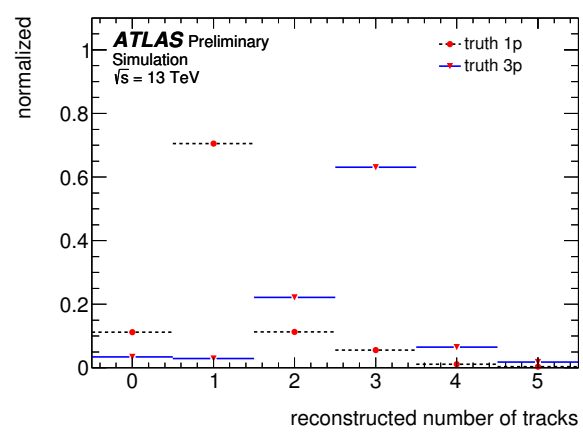

(b) Track reconstruction

Figure 1: (a) Efficiency for correct production vertex assignment in 1-prong tau decays for the tau reconstruction algorithm and the default choice of the vertex with the highest $\Sigma p_{\mathrm{T}}^{2}$, as a function the number of reconstructed vertices in the event, and (b) the number of reconstructed tracks for $\tau_{\text {had-vis }}$ candidates from true 1-prong and 3-prong tau decays. [2]

Tracks are associated with the tau if they meet the following criteria: they must be within $\Delta R<0.2$ of the tau jet center, with $p_{\mathrm{T}}>1 \mathrm{GeV}, 2$ Pixel hits (with the Insertable B-Layer), 7 Pixel + Semi-Conductor-Tracker hits, $d_{0}<1.0 \mathrm{~mm}$, and with $\left|z_{0} \sin \theta\right|<1.5 \mathrm{~mm}$. This selection is designed to maximize the fraction of 1-prong and 3-prong taus reconstructed with the correct number of tracks. The leading source of underestimation of tracks is from tracking inefficiency 
due to hadronic interactions in the inner detector. The leading source of overestimation of tracks is from photon conversion tracks that fulfill the track selection criteria.

\section{Identification}

A Boosted Decision Tree (BDT)-based identification is used to distinguish taus from other sources of hadronic jets.

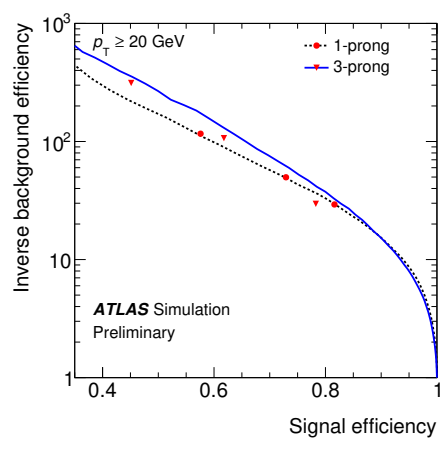

(a) Jet rejection

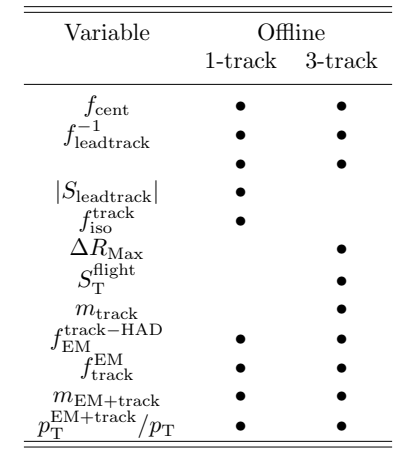

(b) Tau ID variables

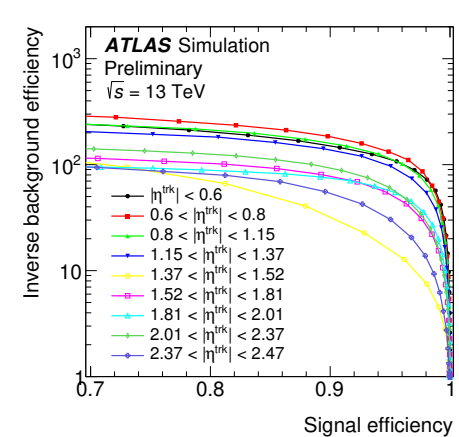

(c) Electron rejection

Figure 2: (a) Inverse of the efficiency for mis-tagging QCD jets as a function of the identification efficiency for $\tau_{\text {had-vis }}$ candidates. The two lines refer to 1-track and 3-track candidates. The EM Loose, EM Medium, and EM Tight working points are shown on these lines. The working points do not stay exactly on the line because they implement variable cuts to achieve a reduced $p_{\mathrm{T}}$-dependentcy of the efficiency. (b) Discriminating variables used as input to the tau identification algorithm at offline reconstruction and at trigger level, for 1-track and 3-track candidates. The bullets indicate whether a particular variable is used for a given selection. The $\left|S_{\text {leadtrack }}\right|$ and $S_{\mathrm{T}}^{\text {flight }}$ variables are calculated with respect to the beam line in the trigger. (c) The inverse of electron mis-identification efficiency as a function of the tau identification efficiency for different $|\eta|$ regions. The nominal working point corresponds to a tau identification efficiency of 95\%. [2]

Working points are defined for 60 (50), 55 (40) and 45 (30) \% reco+ID efficiency for 1-prong (3-prong) taus, with efficiencies approximately constant vs $p_{\mathrm{T}}$ and pileup. Separate BDTs are trained for 1-prong and 3-prong taus on $Z / \gamma \rightarrow \tau \tau$ signal and dijet backgrounds. Identification (ID) variables constructed from tracks and topological calorimeter clusters (topoclusters) in the $\Delta R<0.2$ core region and corrected for pileup. Electron ID is relevant to the discrimination between electrons and taus. If an electron is found within $\Delta R<0.4$ of a tau, with a log-likelihood (LLH)based electron ID score above threshold, then the tau is rejected. The nominal working point for electron rejection is defined for $95 \%$ tau ID efficiency.

\section{Energy Calibration}

Tau Energy Scale (TES) calibration applies a correction of the form $E_{\text {calib }}=\frac{E_{\mathrm{LC}}-E_{\text {pileup }}}{R\left(E_{\mathrm{LC}}-E_{\text {pileup }}, \eta, n_{p}\right)}$, where $E_{\mathrm{LC}}$ is uncalibrated energy, and $n_{p}$ is the number of prongs in the tau decay. $E_{\text {pileup }}$ is a pileup correction found to be linear with the number of vertices in the event, and $R$ is a function that accounts for variations in detector response due to calorimeter thresholds, and decay products 
not reaching the calorimeters or falling outside the tau cone. This factor is extracted as the Gaussian mean of $\left(E_{\mathrm{LC}}-E_{\text {pileup }}\right) / E_{\text {true }}^{\mathrm{vis}}$, and energy resolution is computed as the Gaussian width of $E_{\text {calib }} / E_{\text {true }}^{\text {vis }}$.

\section{Performance}

Performance of the online (trigger) and offline tau identification and the energy calibration are measured using $Z \rightarrow \tau \tau$ events selected from the data. Semileptonic events, where one tau decays to a muon and the other hadronically, are used to select data events with a high purity of true hadronic decays of taus. Electron rejection is measured in $Z \rightarrow e e$ events.

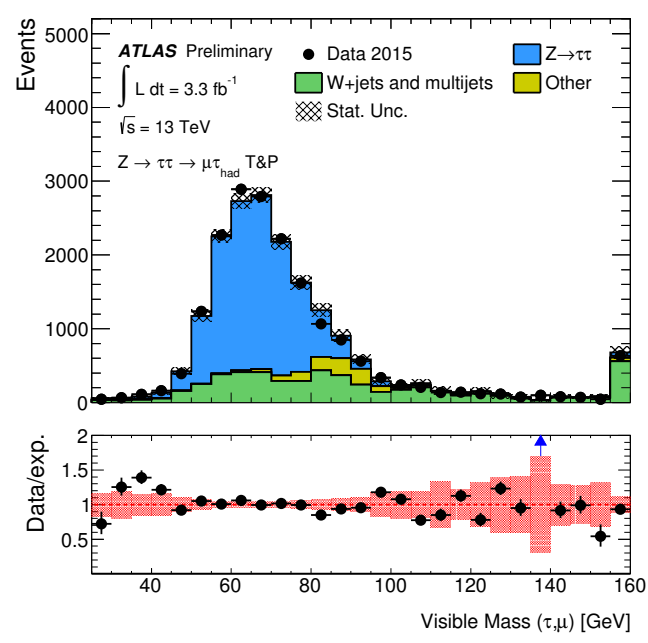

Figure 3: The visible mass reconstructed using isolated muons and offline tau candidates passing the offline loose identification requirement. The $Z$ mass peak is observed in an enriched sample of $Z \rightarrow \tau \tau \rightarrow \mu \tau(\mathrm{had})$ events from the 2016 dataset in $13 \mathrm{TeV}$ collisions, corresponding to an integrated luminosity of $7.1 \mathrm{fb}^{-1}$ [3]. These events are collected using a single-muon trigger. Event selections and background estimations are described in [4]. Only statistical uncertainties are shown.

\section{References}

[1] ATLAS Collaboration, 2008 JINST 3 S08003

[2] ATLAS Collaboration, ATL-PHYS-PUB-2015-045

[3] ATLAS Collaboration, https://twiki.cern.ch/twiki/bin/view/AtlasPublic/TauPublicCollisionPlots

[4] ATLAS Collaboration, ATL-PHYS-PUB-2015-025, Eur. Phys. J. C75 (2015) 303 